\title{
Single-electron charging phenomena in nano/polycrystalline silicon point contact transistors
}

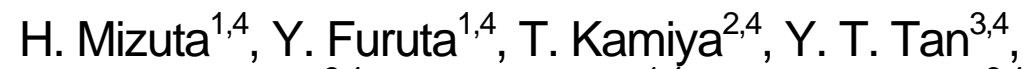 \\ Z. A. K. Durrani ${ }^{3,4}$, K. Nakazato ${ }^{1,4}$ and H. Ahmed ${ }^{3,4}$ \\ ${ }^{1}$ Hitachi Cambridge Laboratory, Hitachi Europe Ltd., Cavendish Laboratory, \\ Madingley Road, Cambridge CB3 OHE United Kingdom \\ ${ }^{2}$ Materials and Structures Laboratory, Tokyo Institute of Technology, 4259 Nagatsuka, \\ Midori-ku, Yokohama 226-8503, Japan \\ ${ }^{3}$ Microelectronics Research Centre, Cavendish Laboratory, University of Cambridge, \\ Madingley Road, Cambridge CB3 OHE, United Kingdom \\ ${ }^{4}$ CREST JST (Japan Science and Technology), Shibuya TK Bldg.,3-13-11 Shibuya, \\ Shibuya-ku, Tokyo 150-0002, Japan
}

Keywords: polycrystalline silicon, grain boundary, single-electron tunnelling, Coulomb blockade, point contact transistor

\begin{abstract}
This paper gives a review of our recent work investigating the physics of single-electron charging phenomena in nano/polycrystalline silicon nanostructures. We first provide a short overview on the research of silicon-based single-electron devices from the last decade. Various single-electron transistor structures are compared in terms of control of electron islands and tunnel barriers. We then study the single-electron charging phenomena in nano/polycrystalline silicon nanostructures. A novel point-contact transistor is introduced, which features an extremely short and narrow nano/poly-Si nanowire as the transistor's channel. This structure is suitable for studying how a grain smaller than $10 \mathrm{~nm}$ in size and a discrete grain boundary work as a charging island and a tunnel barrier, respectively. The relationships between structural and electrical parameters of grains/grain-boundaries and the resulting Coulomb blockade characteristics for the point contact transistors are investigated by applying various passivation processes. Finally, optimisation of grain and grain-boundary structures is discussed for improving the Coulomb blockade characteristics and realizing nano/poly-Si single-electron transistors operating at room temperature.
\end{abstract}

\section{Introduction}

Over the last few decades, the performance of VLSI circuits has been improved steadily by scaling down device dimensions. In dynamic random access memories (DRAMs), for instance, the amount of charge stored per memory cell has been decreased with reduction of the cell area. After the 1 Mbit generation, however, it has become increasingly difficult to keep up such a continuous decrease in the stored charge per bit because the signal becomes less immune to leakage current, internal noise, and soft errors. In microprocessors, power consumption per one MOS transistor has also been reduced due both to miniaturisation and to improved operation conditions. Nevertheless, the total power consumption per microprocessor has gradually been increasing as the number of MOS transistors per chip increases, and the number of electrons used to switch one MOS transistor on and off needs to be reduced further to counter this trend. However, when the number of electrons becomes less than 1000, inherent charge fluctuations cause unacceptable statistical variations in the subthreshold characteristics of the MOS transistors. For both memory and logic applications, how to guarantee future 'scalability' of the devices is a key issue, along with a reduction of the number of electrons. Single- and few-electron devices are expected to overcome these issues by introducing the Coulomb blockade (CB) effect [1],[2] as a new principle for the controlled transfer of a small 
number of electrons.

A key building block for single- and fewelectron devices are the double tunnel junction (DTJ) and multiple tunnel junction (MTJ) structures shown in Figs. 1(a) and (b), which are composed of a series of islands with tunnel barriers between them. When a single electron enters onto the island, the charging energy $E_{C}$ of the island increases, and the transfer of even one electron is strongly suppressed (Coulomb blockade) if the charging energy is higher than

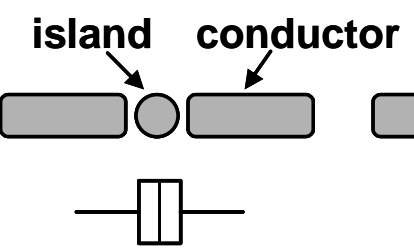

(a)

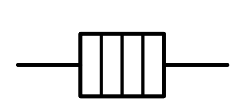

(b)

Fig.1: (a) Double tunnel junction and (b) multiple tunnel junction. the thermal energy $k_{B} T$. From the device-engineering point of view, the MTJ is more preferable than the simple DTJ since it reduces co-tunnelling [3], which generally leads to unfavourable leakage current. Also the MTJ structure is robust against offset charge effects [4][5], which vary the Coulomb gap and even may break the CB. In general, the tunnel junction (TJ) should meet the following two requirements to show the $\mathrm{CB}$ effects at a temperature $T$ :

$$
\begin{aligned}
& R_{t} \gg R_{Q}=h / e^{2}=25.6 \mathrm{k} \Omega \\
& E_{C}=e^{2} / 2 C_{\Sigma} \gg k_{B} T
\end{aligned}
$$

to avoid quantum and thermal smearing of the electronic states confined in the islands. In Eqs. (1) and (2) $R_{Q}$ is the quantum resistance ( $\equiv h / e^{2}$ ), and $R_{t}$ and $C_{\Sigma}$ are the total tunnel resistance of the TJ and total capacitance of the electron islands, respectively.

A number of fabrication methods for DTJs and MTJs have been reported. These structures may be classified into two groups in terms of the manner of formation of nanoscale electron islands: patterned electron islands and naturally formed ones. A silicon-based patterned island structure has been realized by using a pattern-dependent oxidation (PADOX) technique [6]. This technique utilizes faster oxidation caused by stress at the pattern edge, and a DTJ is formed at the both ends of a Si nanowire (NW). This technique has achieved a single electron island with a lateral size of less than $10 \mathrm{~nm}$. An alternative approach is to use an AFM-based oxidation technique [7]. A $\mathrm{NbO}_{2}$ DTJ was defined on a $\mathrm{Nb} \mathrm{NW}$ resulting in a $\mathrm{Nb}$ single island with a lateral dimension of a few $\mathrm{nm}$. A step edge cut off (SECO) technique [8]

has also been reported to form a Table I: Comparison of various methods of singlemetallic MTJ structure.

Naturally formed structures exploit various kinds of local disorder in nanometer-scale structures to form the DTJ or MTJ. In a heavily doped Si NW [9] with a few tens of nanometers in width, randomly distributed dopant atoms cause potential fluctuations, and a linear chain of electron islands are formed when a negative gate bias is applied to a gate electrode placed adjacent to the NW [10]. This is a fairly simple structure and has often been used for making CB memory [11][12] and logic [13] devices. However, the $\mathrm{CB}$ oscillation can usually be observed electron transistor fabrication. Symbols,$\times, \mathbf{A}$ in the

\begin{tabular}{|c|c|c|c|c|c|c|}
\hline & \multicolumn{3}{|c|}{ Naturally-formed } & \multicolumn{3}{|c|}{ Patterned } \\
\hline & $\begin{array}{l}\begin{array}{l}\text { Random } \\
\text { dopant } \\
\text { induced }\end{array} \\
\end{array}$ & $\begin{array}{c}\text { Rough- } \\
\text { ness } \\
\text { induced }\end{array}$ & $\begin{array}{r}\text { Defect } \\
\text { induced }\end{array}$ & $\begin{array}{c}\text { PADOX } \\
\text { based }\end{array}$ & $\begin{array}{c}\text { SPM } \\
\text { based }\end{array}$ & $\begin{array}{l}\text { SECO } \\
\text { based }\end{array}$ \\
\hline $\begin{array}{c}\text { Controllability } \\
\text { of dot size }\end{array}$ & $x$ & $x$ & $x$ & & & \\
\hline $\begin{array}{l}\text { Controllability } \\
\text { of dot position }\end{array}$ & $x$ & $x$ & $x$ & & & \\
\hline $\begin{array}{c}\text { Controllability } \\
\text { of tunnel barrier } \\
\text { thickness }\end{array}$ & $x$ & $x$ & $x$ & $\Delta$ & $\Delta$ & $x$ \\
\hline $\begin{array}{c}\text { Controllability } \\
\text { of tunnel barrier } \\
\text { height }\end{array}$ & $x$ & $x$ & $x$ & $x$ & $\Delta$ & $x$ \\
\hline $\begin{array}{l}\text { Compatibility } \\
\text { with Si-ULSI }\end{array}$ & & & & & $x$ & $x$ \\
\hline
\end{tabular}
table mean 'Yes', 'No', and 'Partly Yes', respectively. 
only at temperatures below $77 \mathrm{~K}$ as the tunnel barriers are not high enough to confine electrons at high temperatures. A surface roughness induced MTJ has also been reported for a very narrow MOS channel [14]. The CB oscillation was observed at room temperature for this structure. A room temperature $\mathrm{CB}$ effect has also been observed very clearly in a carbon nanotube (CNT) in which atomic defects seem to play a role in forming electron islands [15].

These SET structures are summarized in Table 1 from the stand-point of controllability of electron islands and tunnel barriers. The disorder-dependent methods are very useful because CB oscillations can be achieved relatively easily, but it is not possible in these methods to design and control the structural and electrical parameters of MTJs. Among the pattern-controlled methods the PADOX is favorable as the size and position of an electron island can be controlled and it is compatible with Si ULSI technologies. However, key parameters of tunnel barriers such as the barrier height and thickness are not controllable with this method.

Compared with the above technologies, the nano/poly-Si NW structure is very attractive for building $\mathrm{CB}$ devices. An ultra thin poly-Si NW has been used to make a single-electron memory device [16] in which both a nanoscale memory node and a sensing channel can be formed naturally. In the poly-Si film, silicon grains and grain boundaries (GBs) between adjacent islands are supposed to act as electron islands and TJs, respectively. Along the GB an electrostatic potential barrier is formed by carriers captured by trap levels in the band gap. The size of the silicon grains can be controlled to a certain extent via a film thickness and/or process conditions for crystallization, and so the poly-Si nanostructures may enable us to go beyond the limit of lithography for realizing 'tailored' electron islands. The macroscopic properties of the poly-Si film have been studied extensively for applications such as thin film transistors (TFTs) and static random access memories (SRAMs), and various techniques have been established to control the grain size and to improve carrier mobility by optimizing passivation conditions. However, the microscopic properties of discrete GBs have not been studied in detail, and there has been no clear guideline to optimize the grains and GBs for high-temperature SET operation. In this work, we examine the structural and electrical properties of the individual grains and GBs as an electron island and a tunnel barrier of a SET by adopting a point-contact transistor (PC-Tr) structure. In addition, we examine the relationship between properties of GB-TJs and the CB characteristics of the PC-Trs by changing the process conditions for achieving room temperature SET operation.

\section{Single-electron charging phenomena in polycrystalline silicon point contact transistors}

Our nanometer-scale PC-Tr features a channel with both the length $L$ and width $W$ as small as the grain size $L_{\text {grain }}$ of the nano/poly-Si film. The electric characteristics for the PC-Trs must therefore reflect the properties of individual grains and GBs contained in the channel. Figure 2 shows a SEM image of a fabricated PC-Tr. The PC-Trs were fabricated in a 50 nm-thick solidphase-crystallized (SPC) poly-Si film: a process

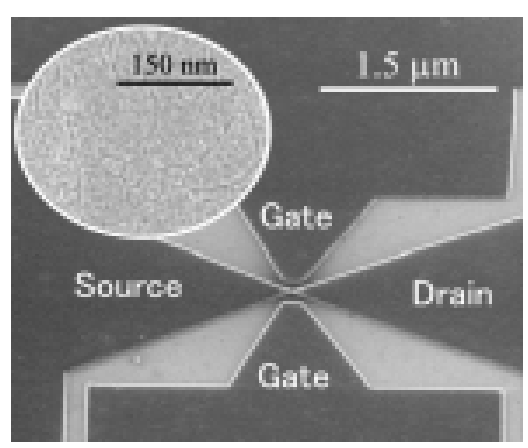

Fig.2: SEM image of an as-prepared point-contact transistor. The inset shows the grain structure of Seccoetched poly-Si film.

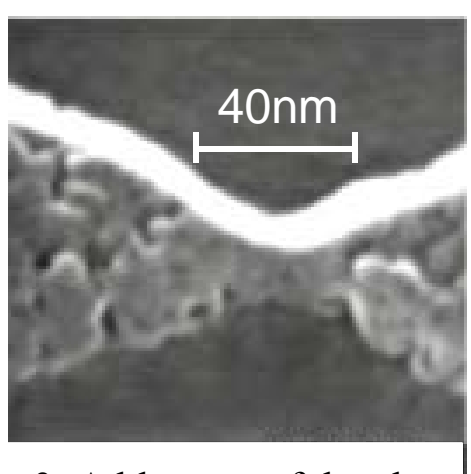

Fig.3: A blow-up of the channel region of the point-contact transistor. 
4. The inset to Fig. 2 is a SEM image of a Seccoetched poly-Si film that shows the individual grains and GBs. From the SEM observation over the large area it was found that $L_{\text {grain }}$ in this particular film ranges from $20 \mathrm{~nm}$ to $150 \mathrm{~nm}$. PC-Trs with a channel width $W$ and length $L$ from $30 \mathrm{~nm}$ to $50 \mathrm{~nm}$ were patterned by a high-resolution e-beam lithography system and electrically isolated by reactive ion etching (RIE). The channel of these PC-Trs is therefore expected to contain only few grains at most [17]. We fabricated more than sixty PC-Trs using the same process. As shown in Fig. 4, half of the PC-Trs were then oxidized at $1000{ }^{\circ} \mathrm{C}$ for $15 \mathrm{~min}$ in dry $\mathrm{O}_{2}$ ambient to study the effects of oxidation on the grains and GB-TJs [18].

Electrical characterization was performed for both as-prepared and oxidized PC-Trs at temperatures down to $4.2 \mathrm{~K}$. An effective potential barrier height $q V_{\mathrm{B}}$ of GBs was extracted from a temperature dependence of the $I_{\mathrm{ds}}-V_{\mathrm{ds}}$ characteristics at between $200 \mathrm{~K}$ and $300 \mathrm{~K}$ using the thermionic emission model [19]:

$$
J=2 q n \sqrt{\frac{k_{B} T}{2 m^{*} \pi}} \exp \left(-\frac{q V_{B}}{k_{B} T}\right) \sinh \left(\frac{q V_{D S}}{2 k_{B} T N}\right)
$$

where $J$ is the current density, $n$ the electron density, $m^{*}$ the electron effective mass, $q V_{\mathrm{B}}$ the potential barrier height of GBs, and $N$ the number of GBs in the channel. Structural characterization was also conducted using TEM and SEM. Figures 5(a) and (b) show two typical $I_{\mathrm{ds}}-V_{\mathrm{ds}}$ characteristics observed for as-prepared PCTrs. The CB effect was not observed for any of the as-prepared PC-Trs at temperatures above $4.2 \mathrm{~K}$. We observed nonlinear $I_{\mathrm{ds}}-V_{\mathrm{ds}}$ characteristics (Fig. 5(b)) associated with electron thermionic emission current over GBs for approximately one third of the asprepared PC-Trs. The other devices showed linear $I_{\mathrm{ds}}-V_{\mathrm{ds}}$ characteristics (Fig. 5(a)). The as-prepared PC-Trs with the nonlinear $I_{\mathrm{ds}}-V_{\mathrm{ds}}$ characteristics would contain a few GBs with a relatively high potential barrier, while those with linear $I_{\mathrm{ds}}-V_{\mathrm{ds}}$ characteristics would have either no GB or a few GBs with a very low potential barrier [17].

In contrast, most of the oxidized PC-Trs show a clear current oscillation in the $I_{d s^{-}} V_{g s}$ curves and a CB gap in the $I_{\mathrm{ds}}-V_{\mathrm{ds}}$ characteristics associated with the CB effect $V_{T}$ : Figs. 6(a) and (b) show typical $I_{\mathrm{ds}}-V_{\mathrm{gs}}$ characteristics for the oxidized PC-Trs measured at $5 \mathrm{~K}$ [18]. We found that the CB characteristics for the oxidized PC-Trs can be classified into two groups: the characteristic with a Coulomb gap $V_{\mathrm{T}}<5 \mathrm{mV}$ and that with $V_{\mathrm{T}}>5 \mathrm{mV}$. The conductance oscillation periods were quite similar among PC-Trs with the same channel dimensions and $V_{\mathrm{T}}<5 \mathrm{mV}$. On the other hand, the oscillation periods differ largely among oxidized PC-Trs with $V_{\mathrm{T}}>5 \mathrm{mV}$. Peak-to-valley (P/V) 
current ratios and tunnel resistances obtained for PC-Trs with $V_{\mathrm{T}}>5 \mathrm{mV}$ were generally much larger than those with $V_{\mathrm{T}}<$ $5 \mathrm{mV}$.

Figures 7(a) and (b) show TEM images of the as-prepared and the oxidized poly-Si films. Both poly-Si films consist of columnar-shaped grains. Temperature dependences of the resistance are shown in Fig. 8. Regarding the as-prepared PC-Trs, although each GB and grain may act as a tunnel barrier and an electron island, the estimated tunnel capacitance is larger than 80 aF because of the tall columnar grains. Also the tunnel resistance $R_{t}$ estimated for

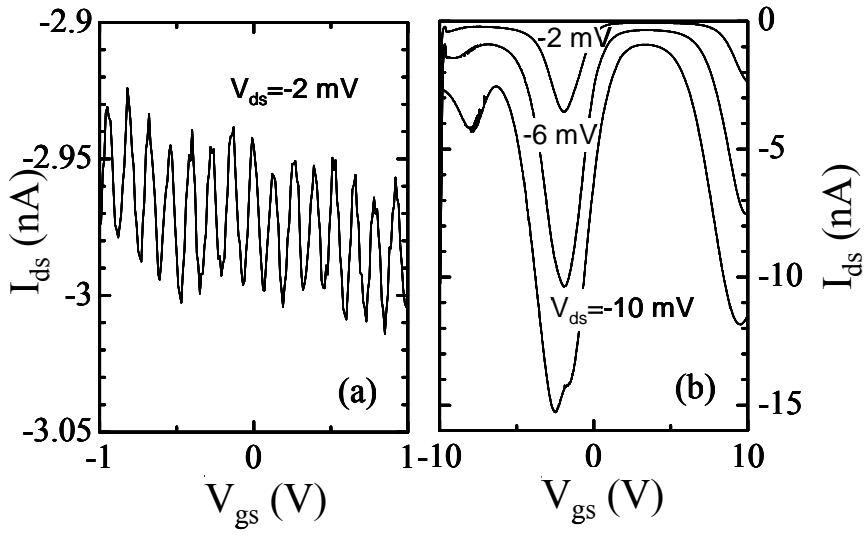

Fig.6: $I_{\mathrm{ds}}-V_{\mathrm{ds}}$ characteristics for oxidised pointcontact transistors with (a) $V_{\mathrm{T}}<5 \mathrm{meV}$ (b) $V_{\mathrm{T}}>$ $5 \mathrm{meV}$. the as-prepared PC-Trs is just as large as the quantum resistance $R_{Q}$ of $25.6 \mathrm{k} \Omega$. Therefore, for the as-prepared PC-Trs, both the electron charging energy $E_{C}$ and the tunnel resistance $R_{t}$ are not sufficiently large to observe the $\mathrm{CB}$ effect even at a cryogenic temperature (see Eqs (1) and (2)). In contrast, in the oxidized PC-Trs, the polySi film thickness decreased down to approximately $18 \mathrm{~nm}$ after the oxidation process [20]. The reduction of the film thickness leads to a decrease in the tunnel capacitance and consequently increases the charging energy. In addition, the tunnel resistance is increased by more than two orders of magnitude after the oxidation treatment, as shown in Fig. 8. Both the increased charging energy and tunnel resistance result in the appearance of the $\mathrm{CB}$ effect as the conditions in Eqs. (1) and (2) are satisfied.

Figure 9 presents the distributions of $q V_{\mathrm{B}}$ obtained for (a) the as-prepared and (b) the oxidized PC-Trs. It is
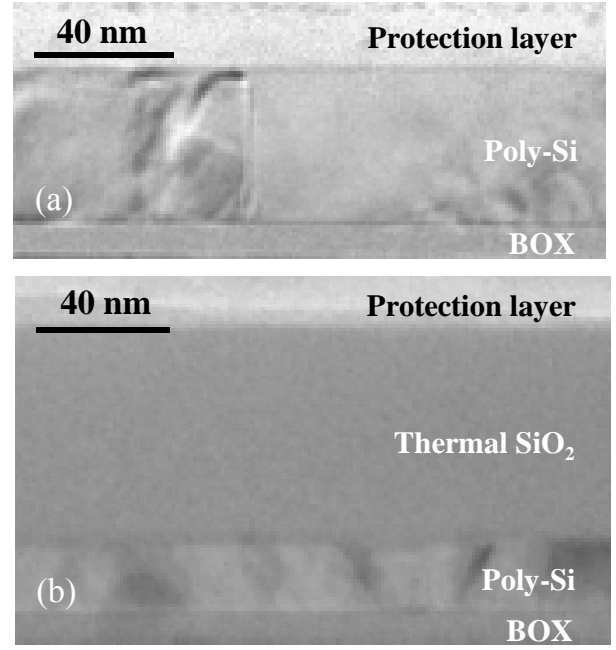

Fig.7: TEM images for (a) as-prepared and (b) oxidized poly-Si films.

found that the mean $q V_{\mathrm{B}}$ increases slightly after the oxidation process. We first note that the oxidized PC-Trs with $V_{\mathrm{T}}<5 \mathrm{mV}$ show a narrow distribution of $q V_{\mathrm{B}}$ (white bars in Fig. 9(b)) which resembles that for the as-prepared linear $I_{\mathrm{ds}}-V_{\mathrm{ds}}$ PC-Trs (white bars in Fig. 9(a)). On the other hand, $q V_{\mathrm{B}}$ for the oxidized PC-Trs with $V_{\mathrm{T}}>5 \mathrm{mV}$ spreads widely (dark gray bars in Fig. 9(b)), corresponding to the distribution of $q V_{\mathrm{B}}$ for the as-prepared PC-Trs with the nonlinear $I_{\mathrm{ds}}-V_{\mathrm{ds}}$ (dark gray bars in Fig. 9(a)). These distributions represent unoxidized and oxidized GBs in the channel, and $q V_{\mathrm{B}}$ can vary a lot depending on the number and configuration of GBs in the individual devices.

As discussed earlier, it is thought for as-prepared PC-Trs with linear $I_{\mathrm{ds}}-V_{\mathrm{ds}}$ characteristics (Fig. 5(a)), that the device incorporated a grain larger than the size of the NW channel and there was no GB in the channel

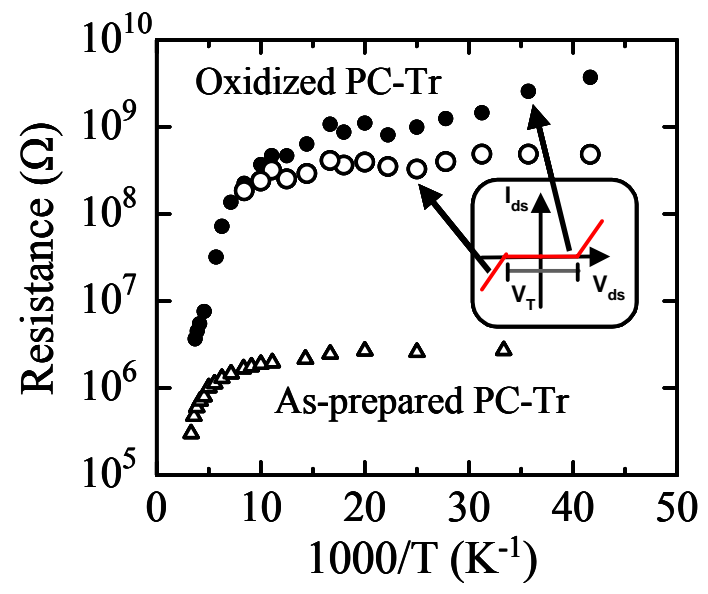

Fig.8: Resistance vs. 1/T for the asprepared and oxidised point-contact transistors. 
to affect the electron transport. All the GBs are contained in the large contact regions and would only show their averaged potential barrier heights. Under these circumstances, the lengthy GBs in the contact regions are not oxidized entirely. Another possibility is that there exist some GBs in the NW channel that do not have many defect states and therefore do not form high potential barriers. The oxidation speed along such low-defect GBs is not very fast and does therefore not have a large effect on their barrier properties. On the other hand, the GBs with larger $q V_{\mathrm{B}}$ obtained for the as-prepared PC-Trs with the nonlinear $I_{\mathrm{ds}}-V_{\mathrm{ds}}$ characteristics are thought to contain large defect densities. These GBs are oxidized effectively [21] and converted to the suboxide tunnel barriers with relatively high barrier heights.

The GB tunnel barrier thickness $d$ was estimated for the oxidized PC-Trs using a tunneling current simulator based on the transfer-matrix method [22]. In the simulation the tunnel barrier was assumed to be a simple rectangular shape with the barrier thickness $d$ and the barrier height obtained as above, and the tunnel resistance of the rectangular barrier was calculated for various values of $d$. By comparing the observed tunnel resistance with the simulated one, the GB barrier thickness $d$ of approximately $3 \mathrm{~nm}$ was obtained for PC-Trs with $V_{\mathrm{T}}>5 \mathrm{mV}$. This is consistent with highresolution TEM observation which reveals that GBs were oxidized faster than crystalline grains and that some silicon suboxide layers are as thick as about $3 \mathrm{~nm}$. This result also supports the view that the CB characteristics for PC-Trs with $V_{\mathrm{T}}>5 \mathrm{mV}$ are determined by the selectively oxidized GBs in the channel.

The dimension of the grain that acts as a charging island was evaluated by using a twodimensional capacitance calculation [23] and the observed Coulomb gap. First, for the oxidized PCTr with $V_{\mathrm{T}}<5 \mathrm{mV}$ corresponding to Fig. 6(a), the charging island size was estimated to be about 30 $\mathrm{nm}$; a very similar island size was obtained for all the other PC-Trs with $V_{\mathrm{T}}<5 \mathrm{mV}$. This figure is similar to the channel dimensions, $L$ and $W$, and it appears that the entire channel region acts as a charging island in these devices. The DTJ could be formed by the oxidised GBs that are outside the channel but still adjacent to the channel edges. It is, however, more likely that the DTJ is formed by the PADOX-mode oxidation [24]. This scenario is supported by the fact that the CB oscillation periods $\Delta V_{g s}$ obtained for the these PC-Trs with $V_{\mathrm{T}}<5 \mathrm{mV}$ are quite uniform among the fabricated devices and also show a dependence on the channel length $L: \Delta V_{g s}=0.57 \pm 0.1 \mathrm{~V}$ for $L=30 \mathrm{~nm}$ and $\Delta V_{g s}=0.18 \pm 0.04 \mathrm{~V}$ for $L=40 \mathrm{~nm}$. In contrast, the island size estimated for the oxidized PCTr with $V_{\mathrm{T}}>5 \mathrm{mV}$ shown in Fig. 6(b) is as small as about $3 \mathrm{~nm}$. This result is also consistent with the above discussion that few GBs existing in the channel were oxidized, which form very small electron charging islands. 
Figure 10 shows the Coulomb gap $E_{C}$ and the $\mathrm{P} / \mathrm{V}$ current ratio as a function of the tunnel resistance $R_{t}$ obtained for the oxidized PC-Trs at $4.2 \mathrm{~K}$. It shows that both the $\mathrm{P} / \mathrm{V}$ ratio and the Coulomb gap increase with increasing tunnel resistance. It can be seen that $R_{t}>1 \mathrm{M} \Omega$ is needed to obtain good $\mathrm{P} / \mathrm{V}$ ratios. The larger Coulomb gap results from an increase in the tunnel resistance and a decrease in the tunnel capacitance due to the formation of thicker GB tunnel barriers with the oxidation process. For improving the $\mathrm{CB}$ characteristics of the poly-Si PC-Trs, the structural parameters of GBs, such as $q V_{\mathrm{B}}$ and $d$, could be optimized further through various oxidation, annealing and passivation treatment as discussed in the next section.

\section{Optimisation of grain and grain boundaries towards room-temperature device operation}

From the above results, we may have a guideline of optimizing the grain/GB parameters for further improvement of the CB characteristics beyond the general conditions in Eqs. (1) and (2). Regarding the grain size, we found that

$$
L_{\text {grain }} \lesssim 5 \mathrm{~nm}
$$

is needed for achieving a large $\mathrm{P} / \mathrm{V}$ current ratio. In addition, we saw that for the tunnel resistance, $R_{t}>1 \mathrm{M} \Omega$ should be achieved. If we assume $L_{\text {grain }} \simeq 3 \mathrm{~nm}$ and $d \simeq 1 \mathrm{~nm}$, a simple calculation [25] shows that

$$
q V_{B} \gtrsim 260 \mathrm{meV}\left(\simeq 10 k_{B} T\right) .
$$

To reduce $L_{\text {grain }}$ overall, a nanocrystalline (nc) Si film prepared by a low-temperature very high frequency (VHF) plasma-enhanced chemical vapor deposition (PECVD) [26][27] was adopted instead of the SPC poly-Si films. A 20nm thick nc-Si:H film was prepared from a $\mathrm{SiF}_{4}: \mathrm{H}_{2}: \mathrm{SiH}_{4}$ gas mixture at temperatures $\leq$ $300{ }^{\circ} \mathrm{C}$. The crystalline volume fraction was estimated to be about $70 \%$ by using Raman spectroscopy. The films were deposited on a $150 \mathrm{~nm}$ thick silicon oxide layer thermally grown on n-type crystalline silicon. The flow rates of the $\mathrm{SiF}_{4}, \mathrm{H}_{2}$ and $\mathrm{SiH}_{4}$ were 30,40 , and $0.25 \mathrm{sccm}$. The VHF frequency was $100 \mathrm{MHz}$, the VHF power was $40 \mathrm{~W}$, and the reactor

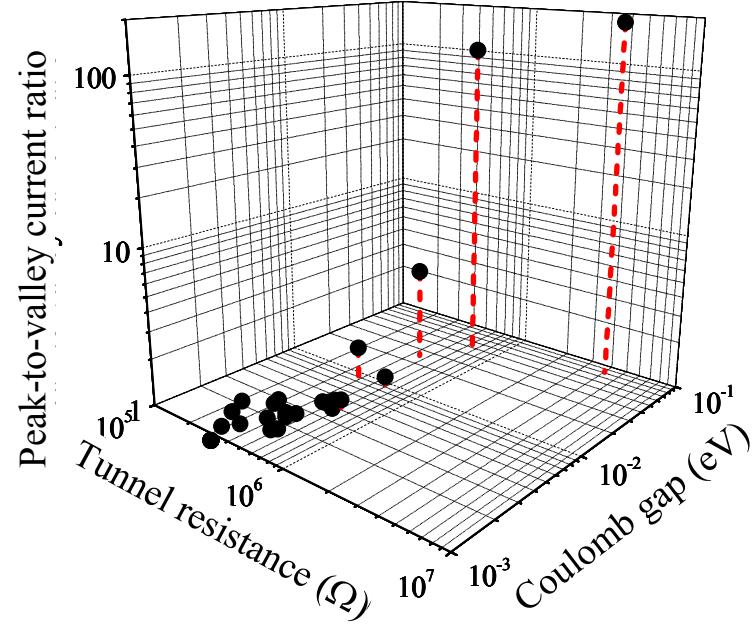

Fig. 10: Coulomb gap and $\mathrm{P} / \mathrm{V}$ current ratio as a function of tunnel resistance. 
pressure was 200 mTorr. As seen in a TEM image in Fig. 11(a), nc-Si grains with $L_{\text {grain }}$ from $4 \mathrm{~nm}$ to $8 \mathrm{~nm}$ are uniformly distributed in an amorphous silicon matrix. PC-Trs were defined in the asdeposited nc-Si film in the same manner as before.

With regard to the condition in Eq. (5) a multiple step oxidation process using low-temperature oxidation $\left(650^{\circ} \mathrm{C}-750{ }^{\circ} \mathrm{C}\right)$ followed by high-temperature $\left(1000^{\circ} \mathrm{C}\right)$ annealing was applied after defining the PC-Trs. In the as-deposited nc-Si:H film, the tunnel barriers are formed by the a-Si:H layer between the grains. The multi-step oxidation process was proposed in order to oxidize the a$\mathrm{Si}: H$ selectively and to convert it to $\mathrm{SiO}_{\mathrm{x}}(\mathrm{x} \ll 2)$ without increasing $L_{\text {grain }}$ [28][29]. It was found from SIMS depth profile of oxygen concentration [29] that the low temperature oxidation step oxidizes the GBs selectively and the subsequent high-temperature annealing increases $q V_{\mathrm{B}}$ and $R_{t}$ of the GBs. Also, encapsulation of the grains with the silicon oxide may prevent any grain growth during high-temperature annealing. This was confirmed by a cross-sectional TEM after the multi-step oxidation process: the grains remained the same in lateral size despite the fact that the film thickness was reduced by 6 $\mathrm{nm}$ due to surface oxide formation (see Fig. 11(b)).

We extracted $q V_{\mathrm{B}}$ for the nc-Si PC-Tr with $\mathrm{L}=\mathrm{W}=20 \mathrm{~nm}$ from a slope of straight lines in the Arrhenius plots of conductivity at a high temperature regime in the same manner as

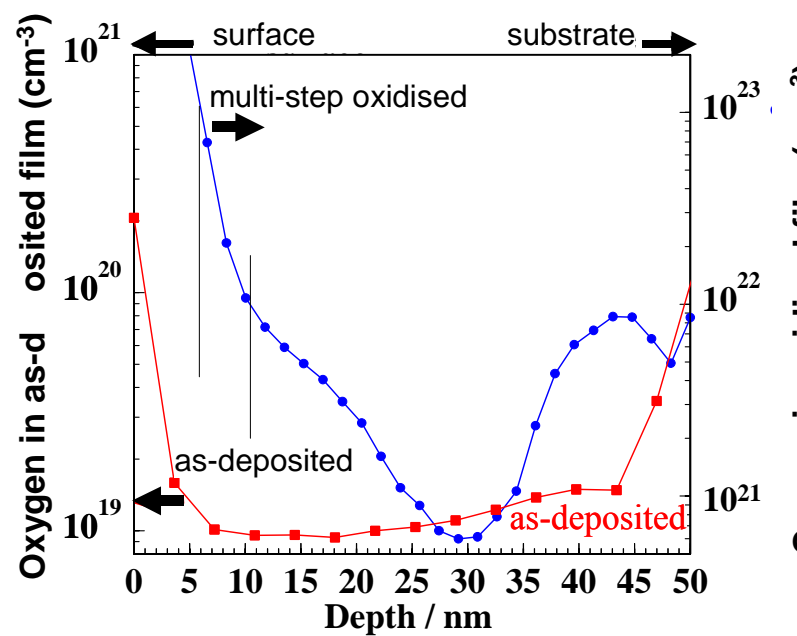

Fig.12: SIMS depth profile of oxygen concentration measured for the as-deposited and multi-step oxidised nc-Si films. before. While the as-deposited nc-Si PC-Trs showed $q V_{\mathrm{B}}$ of about $40 \mathrm{meV}$ which is as low as those obtained for the SPC PC-Trs, the multi-step oxidized PC-Trs showed $q V_{\mathrm{B}}$ of $173 \mathrm{meV}$ that is now much larger than $k_{B} T$ although it does not meet the condition of Eq. (5) completely. The mechanism of the tunnel barrier formation in the multi-step oxidized PC-Trs was investigated carefully by using PC-Trs with channel width $W$ from $20 \mathrm{~nm}$ to $50 \mathrm{~nm}$. It was found that the CB nonlinearity in the $I_{\mathrm{ds}}-V_{\mathrm{ds}}$ characteristics is much weaker for PC-Trs with a larger $W$, implying that the oxygen diffusion from the sidewall of the NW channel is supposed to be as important as that from the upper surface. The SIMS depth profiles of oxygen concentration are shown in Fig. 12 for the as-deposited and multi-step oxidized nc-Si:H films [29]. These results show that the minimum oxygen concentration at the bottom of the 20 -nm-thick nc-Si:H film is about $2 \times 10^{21} \mathrm{~cm}^{-3}$, so that the value of $x$ in $\mathrm{a}_{-} \mathrm{SiO}_{\mathrm{x}}$ is estimated to be only about 0.13 , assuming that the a-Si:H phases with a volume fraction of $30 \%$ were oxidized. If we assume that oxidation also proceeds from both sidewalls and the oxygen concentration is simply given by an addition law, the minimum oxygen concentration at the center of the channel would be $1 \times 10^{22} \mathrm{~cm}^{-3}$, corresponding to $x=0.67$ [29]. As $W$ is increased, this value decreases gradually, resulting in a lower tunnel barrier at the center of the channel.

Figures 13(a) and (b) show the $I_{\mathrm{ds}}-V_{\mathrm{gs}}$ and $I_{\mathrm{ds}}-V_{\mathrm{ds}}$ characteristics observed at various temperatures for the multi-step oxidized nc-Si PC-Tr with $\mathrm{L}=\mathrm{W}=20 \mathrm{~nm}$ [30]. A clear CB oscillation is seen in Fig. 13(a) with an unchanged period, and the oscillation persists up to room temperature although the $\mathrm{P} / \mathrm{V}$ current ratio is gradually decreased as the temperature increases. From a simple capacitance calculation combined with the observed current oscillation period $\Delta V_{g s}$ and CB gap $E_{C}, L_{\text {grain }}$ associated with the single-electron charging phenomena for this nc-Si PC-Tr was estimated to be about $8 \mathrm{~nm}$, which is in good agreement with the TEM observation of Fig. 11(b). These results prove that our guideline is correct for raising the operating temperature of the device. In order to 
improve the $\mathrm{P} / \mathrm{V}$ current ratio of the $\mathrm{CB}$ oscillation and the uniformity of device characteristics at $\mathrm{RT}$, it is necessary to decrease $L_{\text {grain }}$ further as well as increase $q V_{\mathrm{B}}$ by optimizing the multi-step oxidation process [25] to meet the conditions in Eqs. (4) and (5) fully.
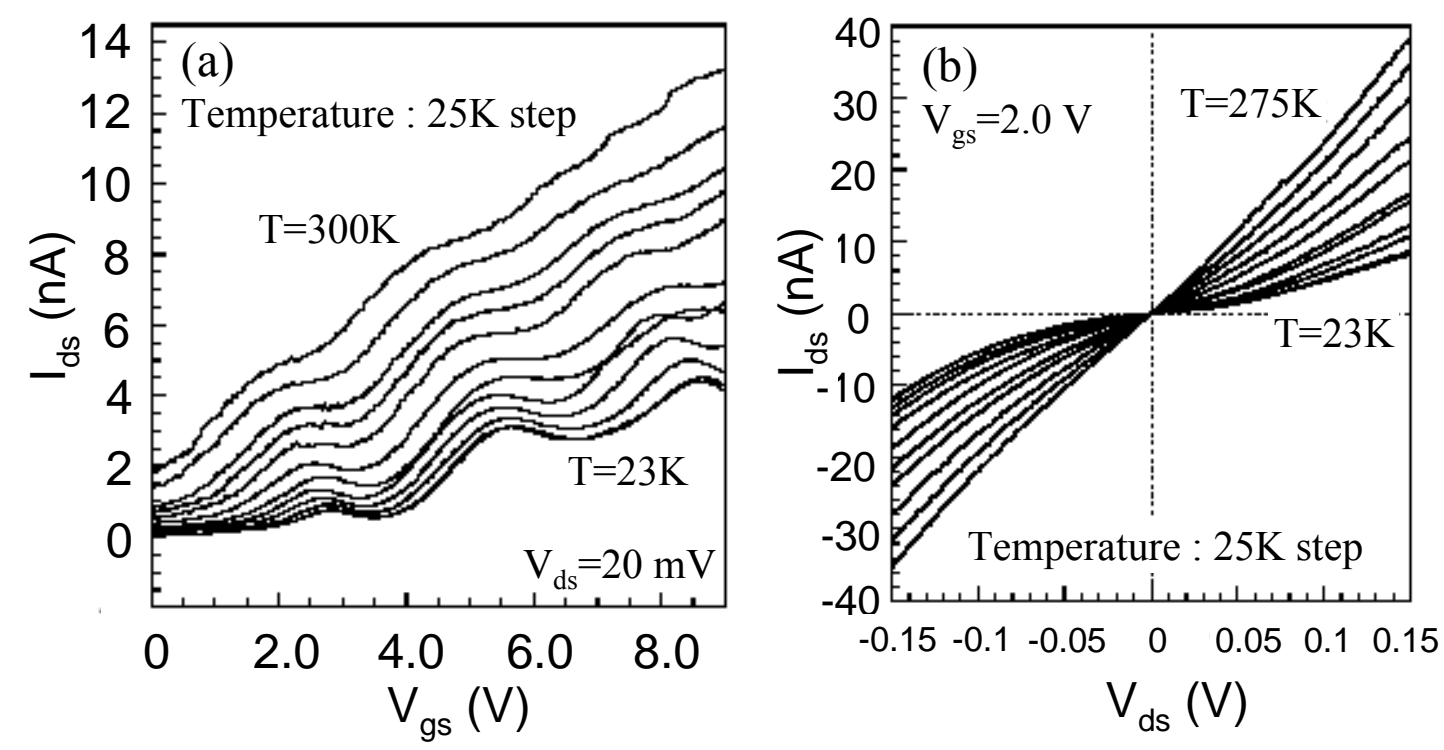

Fig.13: (a) $I_{d s}-V_{g s}$ and (b) $I_{d s}-V_{d s}$ characteristics for the multi-step oxidised nc-Si PC-Tr measured at various temperatures.

\section{Summary}

We have presented our recent work on the single-electron charging phenomena in nano/polycrystalline silicon nanostructures using a PC-Tr structure that features a channel as short as the size of Si grains. First, the electrical and structural characterization of discrete GBs has been performed for as-prepared and oxidized PC-Trs fabricated in a 50-nm-thick heavily doped SPC poly-Si film. No CB oscillation has been observed for the as-prepared PC-Trs at $\mathrm{T} \geqq 4.2 \mathrm{~K}$ mainly because the tunnel resistance of the GBs was not large enough and the tunnel capacitance was too large. By comparison, PC-Trs oxidized at $1000{ }^{\circ} \mathrm{C}$ for 15 min show $\mathrm{CB}$ oscillations at a temperature up to about $40 \mathrm{~K}$ due both to a reduction of the grain height and an increase in the $R_{t}$ of the oxidized GBs. The maximum $q V_{B}$ and $d$ of the oxidized GBs have been found to be $90 \mathrm{meV}$ and approximately $3 \mathrm{~nm}$, respectively. To improve the CB effects, a nc-Si film with $20 \mathrm{~nm}$ thickness fabricated by a low-temperature PECVD has been adopted. The nc-Si film contains smaller Si grains with $L_{\text {grain }}$ from 4 to $8 \mathrm{~nm}$ uniformly distributed in an a-Si matrix. A multi-step oxidation process was applied to the patterned nc-Si PC-Trs to oxidize the GBs more selectively while maintaining $L_{\text {grain }}$. For the multi-step oxidized nc-Si PC-Trs, $q V_{B}$ was found to be as large as 173 $\mathrm{meV}$, resulting in $\mathrm{CB}$ oscillations that persist up to room temperature.

\section{Acknowledgements}

The authors are very grateful to Professor Kenji Taniguchi of Osaka University for his support throughout this work. 


\section{References}

[1] H. Grabert and M. H. Devoret, Eds., Single Charge Tunneling - Coulomb Blockade Phenomena in Nanostructures, NATO ASI Series B (Plenum; New York, 1991).

[2] D.V. Averin and K.K. Likharev, J. Low Temp. Phys. 62, 345 (1986); K. K. Likharev, IEEE Trans. Magn. MAG-23, 1142 (1987).

[3] D.V. Averin and Yu.V. Nazarov, in Ref. [1], 217.

[4] H.-O. Müller, K. Katayama, H. Mizuta, J. Appl. Phys. 84, 5603 (1998).

[5] H.-O. Müller, D. Williams, H. Mizuta, Jpn. J. Appl. Phys. 39, (2000).

[6] Y. Takahashi, H. Namatsu, K. Iwadate, M. Nagase and K. Murase, IEEE Trans. Electron Devices 43, 1213 (1996).

[7] J. Shirakashi, K. Matsumoto, N. Miura and M. Konagai, J. Appl. Phys. 83, 5567 (1998)

[8] S. Altmeyer, A. Hamidi, B. Spangenberg and H. Kurz, J. Appl. Phys. 81, 8118 (1997)

[9] R. Smith and H. Ahmed, J. Appl. Phys. 81, 2699 (1997).

[10] G. Evans, H. Mizuta and H. Ahmed, Jpn. J. Appl. Phys. 40, 5837 (2001).

[11] H.-O. Müller and H. Mizuta, IEEE Trans. Electron Devices 47, 1826 (2000).

[12] Z. Durrani, A. Irvine and H. Ahmed, IEEE Trans. Electron Devices 47, 2334 (2000).

[13] N.J. Stone, and H. Ahmed, Appl. Phys. Lett. 77, 744 (2000).

[14] H. Ishikuro, T. Fujii, T. Saraya, G. Hashiguchi, T. Hiramoto and T. Ikoma, Appl. Phys. Lett. 68, 3585 (1996)

[15] K. Matsumoto, FEMD Newslatter 3, No. 4, 3 (2002)

[16] K. Yano, T. Ishii, T. Hashimoto, T. Kobayashi, F. Murai and K. Seki, IEEE Trans. Electron Devices 41,1628 (1994).

[17] Y. Furuta, H. Mizuta, K. Nakazato, Y. T. Tan, T. Kamiya, Z. A. K. Durrani, H. Ahmed and K. Taniguchi, Jpn. J. Appl. Phys. 40, L615 (2001).

[18] Y. Furuta, H. Mizuta, K. Nakazato, T. Kamiy.a, Y. T. Tan, Z. A. K. Durrani and K. Taniguchi, Jpn. J. Appl. Phys. 41, 2675 (2002)

[19] J. Y. W. Seto, J. Appl. Phys. 46, 5247 (1975).

[20] Y.T. Tan, Z.A.K. Durrani and H. Ahmed, J. Appl. Phys. 89, 1262 (2001).

[21] T. Kamiya, Z.A.K. Durrani and H. Ahmed, to be published in Appl. Phys. Letts. (2002)

[22] H. Mizuta and T. Tanoue, The Physics and Applications of Resonant Tunnelling Diodes (Cambridge University Press, Cambridge, 1995) p. 15.

[23] O.-H. Müller, K. Katayama and H. Mizuta, J. Appl. Phys. 84, 5603 (1998).

[24] T.H.-H. Altebäumer, The Physics and Characterisation of Bi-directional Electron Pump, PhD Thesis 2002, University of Cambridge

[25] T. Kamiya, Y. Furuta, Y.-T. Tan, Z.A.K. Durrani, H. Mizuta and H. Ahmd, Polycrystalline Semiconductors VII - Bulk Materials, Thin Films, and Devices, T. Sameshima, T. Fukui, H.P. Strunk, J.H. Werner eds., in Series 'Solid State Phenomena', Scitech Publ., Uettikon am See, Switzerland, to be published.

[26] T. Kamiya, K. Nakahata, Y. T. Tan, Z.A.K Durrani and I. Shimizu, J. Appl. Phys. 89, 6265 (2001)

[27] Y.-T. Tan, T. Kamiya, Z.A.K. Durrani and H. Ahmed, Appl. Phys. Letts. 78, 1083 (2001)

[28] T. Kamiya, Z.A.K. Durrani and H. Ahmed, Appl. Phys. Lett. 81, 2388 (2002)

[29] T. Kamiya, Y.-T. Tan, Z.A.K. Durrani and H. Ahmed: J. Non-Cryst. Solids 299-302, 405 (2002).

[30] Z.A.K. Durrani, T. Kamiya, Y.T. Tan, H. Ahmed and N. Lloyd, Microelectronic Engineering 63, 267 (2002) 